\title{
Impaired Left Ventricular Longitudinal Function in Idiopathic Pulmonary Arterial Hypertension Children
}

\author{
Iolanda Muntean, Carmen Șuteu, Rodica Togănel \\ Clinic of Pediatric Cardiology, University of Medicine and Pharmacy, Tîrgu Mureș, Romania
}

\section{CORRESPONDENCE}

\section{lolanda Muntean}

Department of Pediatric Cardiology, Institute of Cardiovascular Diseases and Transplantation, University of Medicine and Pharmacy

50 Gheorghe Marinescu St

540136 Tîrgu Mureș, Romania

Tel: +40 744372276

E-mail: iolanda.muntean@gmail.com

\section{ARTICLE HISTORY}

Received: 21 April, 2016

Accepted: 3 June, 2016
Carmen Suteu • Department of Pediatric Cardiology, Institute of Cardiovascular Diseases and Transplantation, University of Medicine and Pharmacy, 50 Gheorghe Marinescu St, 540136 Tîrgu Mureș, Romania, Tel: +40 744372276

Rodica Togănel • Department of Pediatric Cardiology, Institute of Cardiovascular Diseases and Transplantation, University of Medicine and Pharmacy, 50 Gheorghe Marinescu St, 540136 Tîrgu Mures, Romania, Tel: +40 265211595

\begin{abstract}
Background: Pulmonary arterial hypertension is associated with right ventricular dilation and failure. As a result, left ventricular geometry is affected by shifting of the interventricular septum towards the left ventricle. Aim of the study: The aim of the study was to assess the effect of chronic right ventricular pressure overload on left ventricular longitudinal function and synchronicity in idiopathic pulmonary arterial hypertension children, using speckle-tracking echocardiography. Material and methods: We prospectively evaluated 13 children (5 with idiopathic pulmonary arterial hypertension and 8 sex- and age-matched controls) using conventional and speckle-tracking echocardiography and clinical status (WHO functional class) Left ventricular longitudinal strain curve was generated for 17 segments and global left ventricular longitudinal peak systolic strain was calculated. Dyssynchrony index of the left ventricle was determined calculating the standard deviation of time to peak-systolic strain for 12 left ventricular, 6 basal and 6 midventricular segments, from short axis views. Results: Strain imaging showed significantly decreased global left ventricular longitudinal strain and increased dyssynchrony index in idiopathic pulmonary arterial hypertension patients as compared with controls (-16.80 \pm 2.94 vs. $-21.50 \pm 1.60, p=0.003$, and $53.80 \pm 16.72$ vs. $22.25 \pm 6.18, p=0.0001$, respectively). There was a significant correlation between left ventricular longitudinal strain, dyssynchrony index and right ventricular fractional area changes $(r=-0.66, p=0.013$, and $r$ $=-0.72, p=0.005$, respectively), right ventricular myocardial performance index $(r=0.86, p=$ 0.0001 , and $r=0.93, p=0.000$, respectively), and LV eccentricity index $(r=0.82, p=0.001$, and $r$ $=0.93, p=0.000$, respectively) in the study population as a whole. Conclusions: Left ventricular longitudinal systolic strain and synchronicity are impaired in idiopathic pulmonary arterial hypertension children with normal left ventricular ejection fraction.
\end{abstract}

Keywords: left ventricle, longitudinal peak systolic strain, dyssynchrony, idiopathic pulmonary arterial hypertension, children

\section{INTRODUCTION}

Idiopathic pulmonary arterial hypertension (i-PAH) is a rare disease in childhood, with a reported annual incidence of about 0.7 cases per million. ${ }^{1}$ It is associated with significant morbidity and mortality. ${ }^{2,3}$

Right ventricular (RV) function has been identified as an important prognostic factor in patients with PAH. ${ }^{4,5}$ Right ventricular failure and dilation may in- 
terfere with left ventricular (LV) function because of the interventricular septal shift toward the left ventricle.6,7

Speckle-tracking echocardiography is a relatively novel technique that allows the assessment of myocardial deformation, and also ventricular synchronicity. ${ }^{8,9}$

We aim to assess the effect of chronic RV pressure overload on LV longitudinal function and synchronicity in iPAH children, using speckle-tracking echocardiography.

\section{MATERIAL AND METHODS}

This is a prospective clinical observational study. The study protocol was approved by the local Ethics Committee of the University of Medicine and Pharmacy of Tîrgu Mureș, Romania, and written informed consent was given by parents or legal guardian. All the study procedures were performed in accordance with the principles stated in the Declaration of Helsinki.

\section{Study population}

The study group consisted of 13 patients: 5 children with i-PAH on pulmonary vasodilator medication (in monotherapy or combined therapy) and 8 age- and sex-matched healthy children as a control group, with no history of cardiovascular disease (Table 1). All patients underwent a complete physical examination, and conventional and speckle-tracking echocardiographic examination. $\mathrm{PAH}$ was defined as mean pulmonary arterial pressure $>25$ $\mathrm{mmHg}$ at rest ${ }^{1}$. Patients with rhythm disturbances, those with PAH secondary to congenital heart defects (Nice, $2013^{2}$ ) and patients older than 18 years were excluded from the study.

\section{Echocardiographic image acquisition}

All children underwent a complete transthoracic echocardiography study using a iE33 system (Philips Medical
Systems, Best, Netherlands). The frame rate was adjusted to 60 to 100 frames per second. Standard 2D images were acquired in the apical views (2-, 3- and 4- chamber), as well as two parasternal short axis views (basal and midventricular). Three consecutive beats were stored in cineloop format, in order to obtain accurate speckle-tracking analysis of the LV. Conventional echocardiographic parameters such as right ventricular fractional area changes (RV-FAC), tricuspid annular plane systolic excursion (TAPSE), right ventricular myocardial performance index (RV-MPI), tricuspid annular systolic velocity (S') or RV systolic/diastolic (S/D) ratio, mitral annular plane systolic excursion (MAPSE), RV-RA gradient, mean pulmonary arterial pressure (PAPm), LV ejection fraction (LV-EF) and LV eccentricity index (LV-EI) were measured as recommended by current guidelines. ${ }^{8,10}$

\section{Two-dimensional speckle-tracking analysis}

Speckle-tracking analysis of the LV was performed offline using QLAB 10 software (Philips Medical Systems, Best, Netherlands). A seventeen-segment model was created according to the software. LV longitudinal strain curve was automatically generated for every segment. Then apical 2-, 3- and 4 chamber, as well as global LV longitudinal peak systolic strain (LpsS) were automatically calculated. The dissynchrony index of the LV was also determined by calculating the standard deviation of time to peak-systolic strain for $12 \mathrm{LV}$ segments, 6 basal and 6 midventricular, from short axis views $\left(\mathrm{SDt}_{12 \mathrm{~s}}\right)$ (Figure 1 ).

\section{Statistical analysis}

Statistical analysis was performed using SPSS version 20 (IBM SPSS STATISTICS 20). Data were labelled as nominal or quantitative variables. Nominal variables were expressed as number or percentage. Quantitative variables were expressed as mean \pm SD. Differences between two

TABLE 1. Clinical characteristics of i-PAH patients and normal controls

\begin{tabular}{lccc}
\hline Variable & i-PAH $(\mathbf{n}=\mathbf{5})$ & Control $(\mathbf{n}=\mathbf{8})$ & p value \\
\hline Age $(\mathrm{y})$ & $8.76 \pm 2.15$ & $8.97 \pm 2.26$ & 0.86 \\
Male & $3(60 \%)$ & $4(50 \%)$ & 1.000 \\
W $(\mathrm{kg})$ & $25.00 \pm 7.50$ & $31.18 \pm 10.05$ & 0.264 \\
$\mathrm{H}(\mathrm{cm})$ & $126.60 \pm 11.12$ & $136.75 \pm 12.03$ & 0.157 \\
$\mathrm{BSA}\left(\mathrm{m}^{2}\right)$ & $0.93 \pm 0.17$ & $1.07 \pm 0.21$ & 0.232 \\
$\mathrm{BMI}\left(\mathrm{m} / \mathrm{kg}^{2}\right)$ & $15.29 \pm 2.39$ & $16.24 \pm 2.29$ & 0.491 \\
WHO Functional class $1 / 2 / 3 / 4$ & $\mathrm{NA}$ & $0 / 2 / 2 / 1$ & \\
Medication S/B/S+B & $\mathrm{NA}$ & $1 / 1 / 3$ & $\mathrm{NA}$ \\
\hline
\end{tabular}

$\mathrm{B}$ - Bosentan; BMI - body mass index; BSA - body surface area; $\mathrm{H}$ - height; i-PAH - idiopathic pulmonary arterial hypertension; $\mathrm{S}$ - Sildenafil; $\mathrm{W}$ - weight. Variables are expressed as mean \pm SD or as number/percentage. 


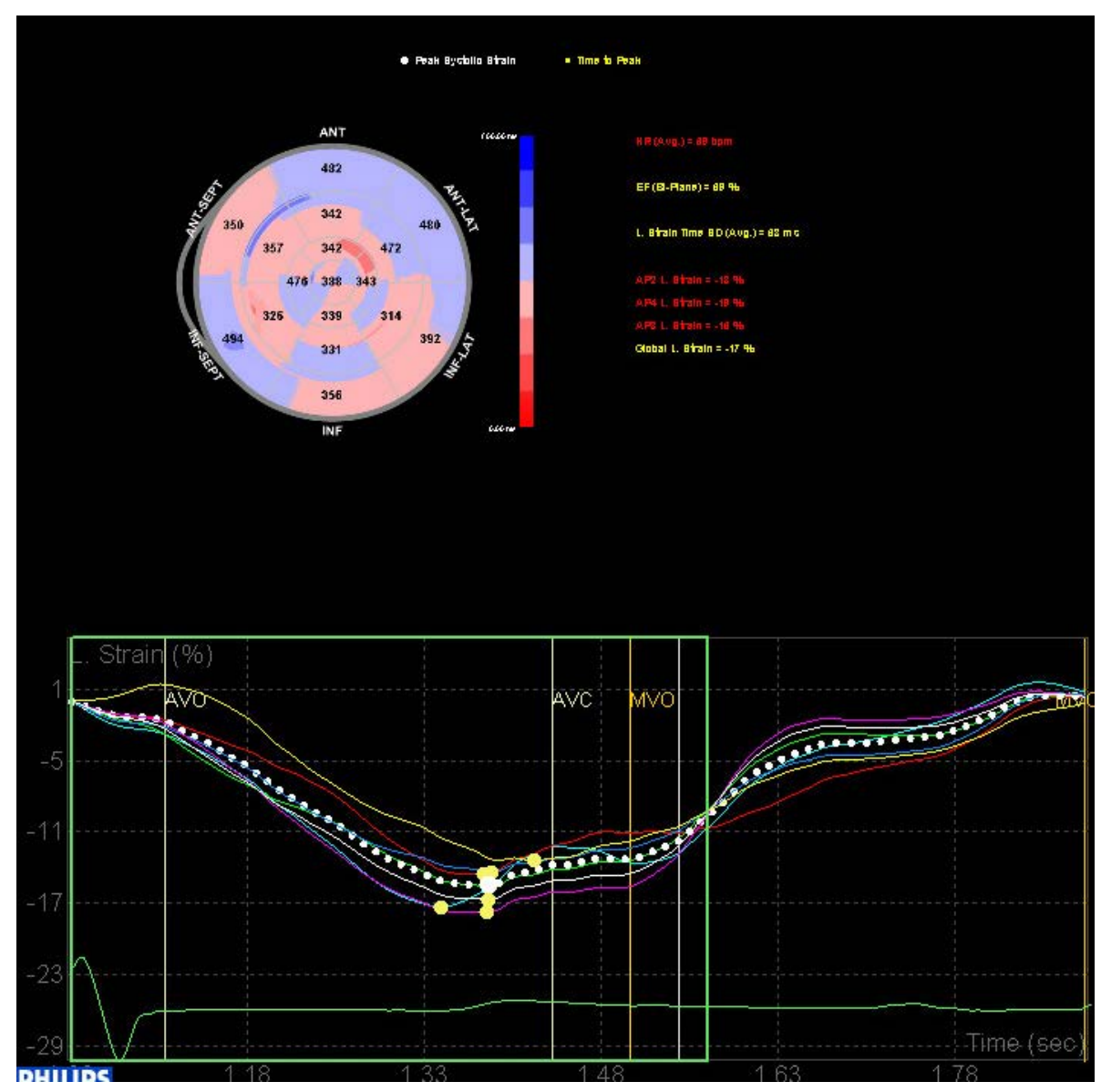

FIGURE 1. Bulls-eye representation of time to peak of systolic strain for 17 left ventricular segments. Strain curves of left ventricular segments.

groups with normally distributed variables were analysed using the unpaired t test. Pearson's correlation coefficient (r) was used to assess correlation between variables. The level of statistical significance was set at $\mathrm{p}<0.05$.

\section{RESULTS}

\section{Clinical characteristics}

The most important clinical characteristics of the study groups are presented in Table 1. There are no significant differences regarding body weight, height, body surface area or body mass index between the two groups $(\mathrm{p}>0.05)$.

\section{Conventional echocardiographic data}

Table 2 shows the conventional echocardiographic data of the patient groups. RV-FAC and S' were significantly lower in i-PAH patients. Conversely, LV-EI and LV end-diastolic diameter, RV-MPI, RV S/D ratio were significantly higher in i-PAH patients compared with controls. There were no differences between the two groups regarding MAPSE and LV-EF.

\section{D-speckle tracking imaging}

Tabel 2 also shows the comparison of LV-LpsS and SDt ${ }_{12 s}$ by LS between normal controls and patient groups. LVA2C LpsS, LV-A3C LpsS, LV-A4C LpsS and LV-GLpsS LpsS were significantly decreased in i-PAH patients as compared with controls $(-16.40 \pm 3.78$ vs. $-21.62 \pm 2.87$, $\mathrm{p}=0.016 ;-6.40 \pm 1.51$ vs. $-21.50 \pm 0.92, \mathrm{p}=0.000 ;-19.20$ \pm 2.28 vs. $-22.00 \pm 1.69, \mathrm{p}=0.027$; and $-16.80 \pm 2.94$ vs. $-21.50 \pm 1.60, p=0.003$, respectively). Furthermore, $\mathrm{SDt}_{12 \mathrm{~s}}$ by LS was significantly increased in i-PAH children compared to controls ( $53.80 \pm 16.72$ vs. $22.25 \pm 6.18, \mathrm{p}=$ 0.0001) (Figure 2).

There was a significant correlation between LV-GLpsS and RV-FAC ( $\mathrm{r}=-0.66, \mathrm{p}=0.013)$, RV-MPI $(\mathrm{r}=0.86, \mathrm{p}=$ $0.0001)$, D2 end-diastolic LV ( $r=-0.74, p=0.003)$, as well as LV-EI $(r=0.82, p=0.001)$ in the study population as a whole. Similarly, we found a significant correlation be- 
TABLE 2. Comparison between study groups by conventional and speckle-tracking echocardiographic parameters (LV longitudinal strain and dyssynchrony parameters)

\begin{tabular}{lccc}
\hline Variable & i-PAH $(\mathbf{n}=\mathbf{5})$ & Control $\mathbf{( n = 8 )}$ & p value \\
\hline RV-RA grd (mmHg) & $111.60 \pm 29.39$ & NA & NA \\
PAPm (mmHg) & $61.00 \pm 18.54$ & NA & NA \\
RV-FAC (\%) & $24.72 \pm 12.11$ & $49.54 \pm 5.23$ & 0,000 \\
RV-MPI & $0.52 \pm 0.18$ & $0.16 \pm 0.07$ & 0.000 \\
TAPSE (cm) & $1.76 \pm 0.70$ & $2.05 \pm 0.43$ & 0.366 \\
RV S/D ratio & $1.59 \pm 0.22$ & $1.02 \pm 0.25$ & 0.002 \\
S (cm/s) & $10.78 \pm 2.53$ & $13.31 \pm 2.03$ & 0.072 \\
LV-El & $1.61 \pm 0.38$ & $1.00 \pm 0.07$ & 0.001 \\
D1 end-diastolic LV (cm) & $4.12 \pm 0.54$ & $3.77 \pm 0.46$ & 0.247 \\
D2 end-diastolic LV (cm) & $2.67 \pm 0.77$ & $3.75 \pm 0.26$ & 0.004 \\
LV-EF & $67.60 \pm 4.72$ & $69.75 \pm 5.52$ & 0.487 \\
MAPSE(cm) & $1.58 \pm 0.45$ & $1.65 \pm 0.24$ & 0.737 \\
LV-A2C LpsS (\%) & $-16.40 \pm 3.78$ & $-21.62 \pm 2.87$ & 0.016 \\
LV-A3C LpsS (\%) & $-16.40 \pm 1.51$ & $-21.50 \pm 0.92$ & 0.000 \\
LV-A4C LpsS (\%) & $-19.20 \pm 2.28$ & $-22.00 \pm 1.69$ & 0.027 \\
LV-GLpsS (\%) & $-16.80 \pm 2.94$ & $-21.50 \pm 1.60$ & 0.003 \\
SDt 12 by LS (ms) & $53.80 \pm 16.72$ & $22.25 \pm 6.18$ & 0.000 \\
\hline
\end{tabular}

A2C - apical two-chamber view; A3C - apical three-chamber view; A4C - apical four-chamber view; D - diameter; FAC fractional area change; GLpsS - global longitudinal peak systolic strain; grd - gradient; i-PAH - idiopathic pulmonary arterial hypertension; LV-EF - left ventricular ejection fraction; LV-EI - left ventricular eccentricity index; MPI - myocardial performance index; PAPm - mean pulmonary arterial pressure; RA - right atrium; RV - right ventricle; S - tricuspid annular systolic velocity; $\mathrm{S} / \mathrm{D}$ ratio - systolic/diastolic ratio; $\mathrm{SDt}_{12 \mathrm{~s}}$ - standard deviation of the time to peak systolic strain of 12 segments; TAPSE - tricuspid annular plane systolic excursion.

tween $\mathrm{SDt}_{12 \mathrm{~s}}$ and RV-FAC ( $\left.\mathrm{r}=-0.72, \mathrm{p}=0.005\right)$, RV-MPI $(\mathrm{r}=0.93, \mathrm{p}=0.000), \mathrm{RV} \mathrm{S} / \mathrm{D}$ ratio $(\mathrm{r}=0.69, \mathrm{p}=0.008), \mathrm{D} 2$ end-diastolic LV ( $r=-0.80, p=0.001)$, as well as LV-EI ( $r=$ $0.93, \mathrm{p}=0.000$ ) in the study cases (Table 3 , Figure 3 ).

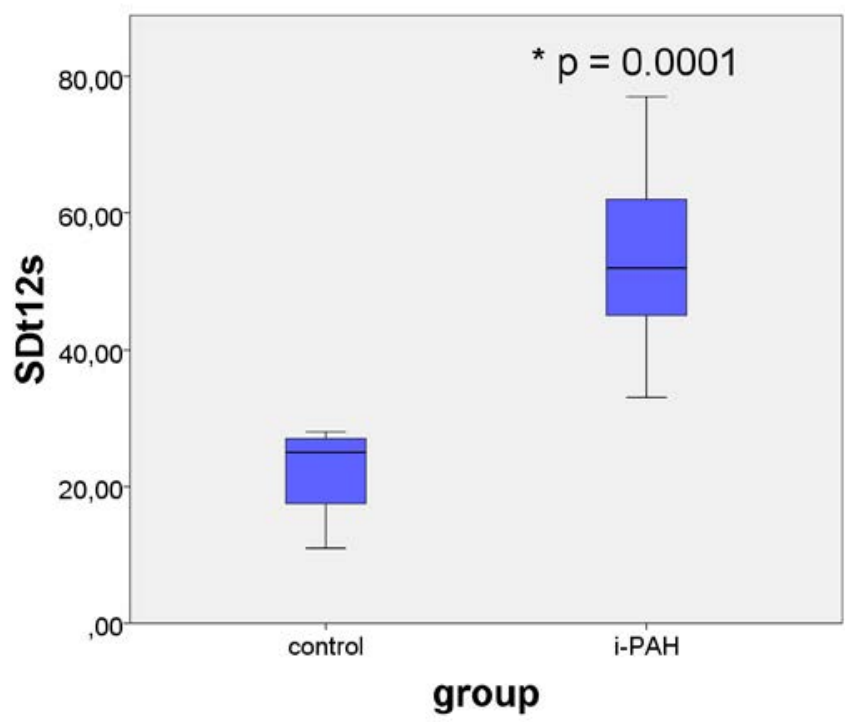

FIGURE 2. Comparison between groups regarding SDt12s by LS: $\mathrm{SDt} 12 \mathrm{~s}$ was significantly increased in $\mathrm{PAH}$ children than in controls; $\mathrm{p}=0.0001$.

i-PAH - idiopathic pulmonary arterial hypertension; $\mathrm{SDt}_{12 \mathrm{~s}}$ - standard deviation of the time to peak systolic strain of 12 segments.

\section{DISCUSSION}

Pulmonary arterial hypertension is associated with an altered right ventricular geometry and function. ${ }^{6}$ Previ-

TABLE 3. Correlation between LV longitudinal strain and dyssynchrony parameters (LV-GLpsS and $\mathrm{SDt}_{12 \mathrm{~S}}$ ) and conventional echocardiographic parameters

\begin{tabular}{lcc}
\hline Variable & $\mathbf{r}$ & p value \\
\hline LV-GLpSS (\%) & & \\
RV-FAC (\%) & -0.66 & 0.013 \\
RV-MPI & 0.86 & 0.000 \\
TAPSE (cm) & -0.61 & 0.027 \\
RV S/D ratio & 0.54 & 0.056 \\
D2 end-diastolic LV (cm) & -0.74 & 0.003 \\
LV-EI & 0.82 & 0.001 \\
SD12S by LS (ms) & & \\
RV-FAC (\%) & -0.72 & 0.005 \\
RV-MPI & 0.93 & 0.000 \\
TAPSE (cm) & -0.49 & 0.089 \\
RV S/D ratio & 0.69 & 0.008 \\
D2 end-diastolic LV (cm) & -0.80 & 0.001 \\
LV-EI & 0.93 & 0.000 \\
D - diameter; FAC - fractional area change; GLpsS - global longitudinal peak systolic strain; \\
LV-EI - left ventricular eccentricity index; MPI - myocardial performance index; RV - right \\
ventricle; S/D ratio - systolic/diastolic ratio; TAPSE - tricuspid annular plane systolic excursion; \\
SDt12s - standard deviation of the time to peak systolic strain of 12 segments
\end{tabular}



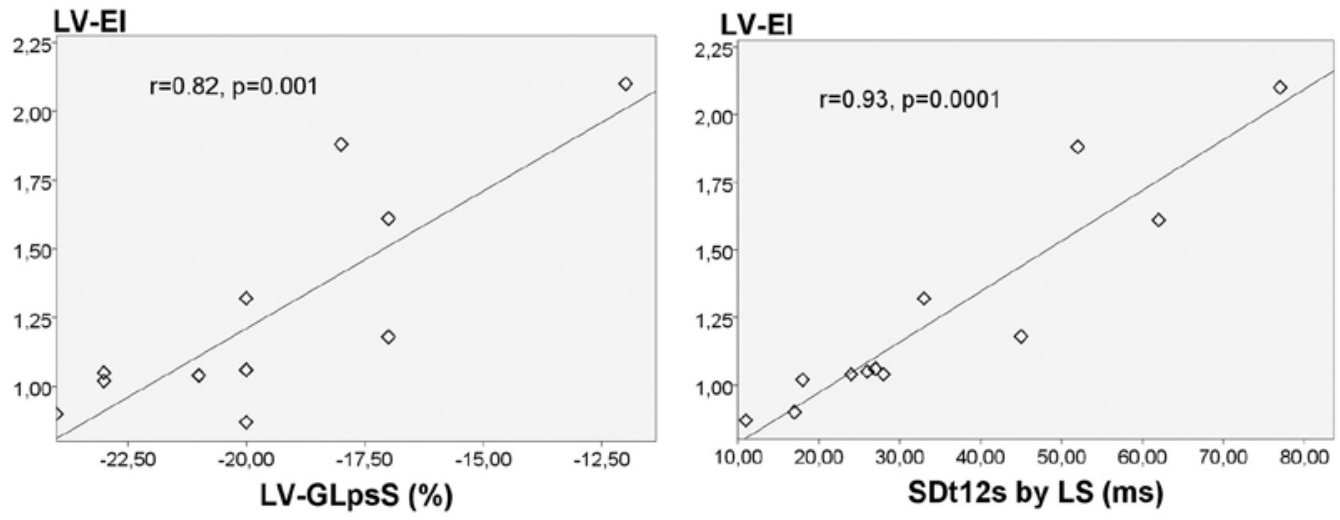

\section{RV-MPI}
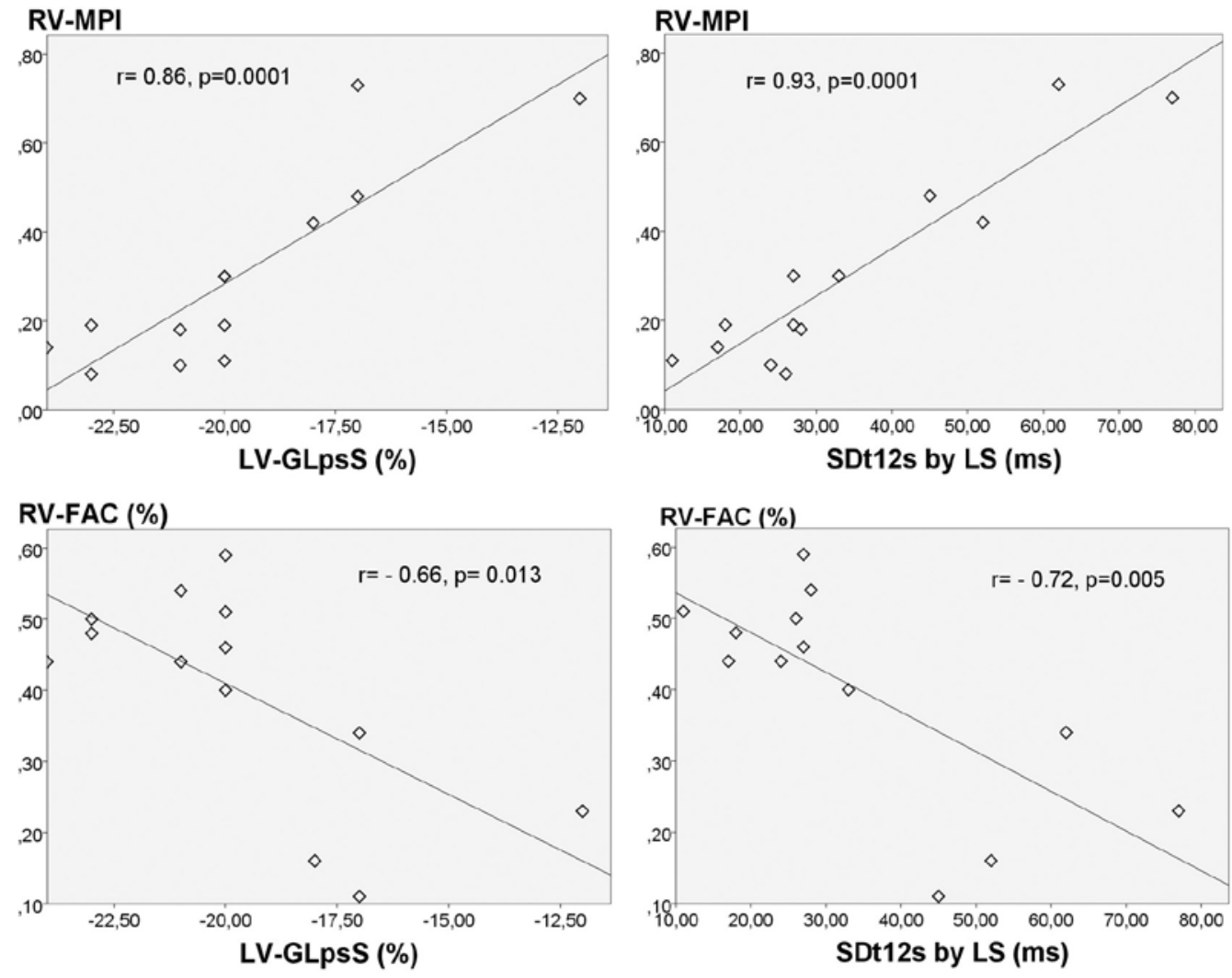

FIGURE 3. Correlation between LV-GLpsS, SDt ${ }_{12 s}$ and LV-EI, RV-MPI and RV-FAC.

FAC - fractional area change; GLpsS - global longitudinal peak systolic strain; LV-EF - left ventricular ejection fraction; LV-EI - left ventricular eccentricity index; MPI - myocardial performance index; RV - right ventricle; SDt ${ }_{12 s}$ - standard deviation of the time to peak systolic strain of 12 segments.

ous studies have reported that, because of the increased pressure, the RV becomes dilated with a more spherical conformation and causes the shifting of the interventricular septum towards the LV. As a result, LV geometry is affected by a decrease of septal-free wall diameter. ${ }^{6,7} \mathrm{In}$ our study we found that end-diastolic LV-EI and also LV end-diastolic septal-free wall diameter (D2) significantly decreased in the i-PAH group compared with controls. Our data confirms the findings of Raymond et al. ${ }^{11}$ and Puwanant et al. ${ }^{12}$, who described increased LV-EI in adult patients with PAH.
LV function can be evaluated on conventional echocardiography by several indices such as EF, MAPSE or S, a tissue Doppler parameter. Two-dimensional speckle-tracking echocardiography is a relatively new imaging technique that could allow myocardial deformation assessment. ${ }^{8}$ This technique has been validated against MRI ${ }^{13}$ and sonomicrometry. ${ }^{14}$ Because of few studies concerned with LV function in PAH children, we conducted this study to evaluate LV global longitudinal strain and LV dyssinchrony in i-PAH children.

Despite normal conventional echocardiographic measures of LV systolic function (EF, MAPSE, S) in the current 
study we found a significantly lower global LV-LpsS in iPAH patients compared with controls. Furthermore, global LV longitudinal strain correlates significantly with RV-FAC, RV-MPI and LV-EI. These findings demonstrate the RV-LV interdependency in PAH setting in children. Previous studies have evaluated LV longitudinal strain in adult patients with PAH. ${ }^{12,15,16}$ Hardegree et al. have de-monstrated in a speckle-tracking study conducted on 71 adult PAH patients, that while conventional LV systolic parameters such as EF remained normal, LV free wall systolic strain was significantly reduced. ${ }^{15}$ Conversely, some studies ${ }^{12,16}$ found significantly decreased interventricular septum longitudinal strain in PAH patients compared to normals, but no difference regarding LV free wall longitudinal strain. However, these latest studies have used tissue Doppler-based instead of 2D speckle-tracking based strain measurements.

LV longitudinal dyssynchrony has been demonstrated in dilated cardiomyopathy with depressed LV function. ${ }^{17,18}$ Also, it has been proved that chronic RV pressure overload is associated with LV radial dyssynchrony. ${ }^{19}$ However, LV longitudinal dyssynchrony has not been studied in children with PAH so far. We found a significantly increased $\mathrm{SDt}_{12 \mathrm{~s}}$ (by LS) in i-PAH children compared to controls, which demonstrates the existence of LV longitudinal dyssynchrony in these children. Furthermore, $\mathrm{SDt}_{12 \mathrm{~s}}$ correlates significantly with RV-FAC, RV-MPI and LV-EI in our study.

Although the present study has proved that LV longitudinal function is impaired in i-PAH children in settings of normal LV-EF, the results have to be validated in larger studies.

\section{Limits of the study}

There was a small number of i-PAH patients, which could be explained by the low incidence of this pathology in the pediatric population. Thus, our observations should be validated in a larger cohort.

\section{CONCLUSION}

Left ventricular longitudinal systolic strain and synchronicity are impaired in idiopathic pulmonary arterial hypertension children with normal left ventricular ejection fraction.

\section{ACKNOWLEDGEMENT}

The present study was carried out in the research project no. 27/11.12.2013, financed by the University of Medicine and Pharmacy of Tîrgu Mureș, Romania.
Thanks to this project, speckle-tracking echocardiography was used for the first time in Romania for the assessment of myocardial deformation in children.

\section{CONFLICT OF INTEREST}

Nothing to declare.

\section{REFERENCES}

1. van Loon RL, Roofthooft MT, Hillege HL, et al. Pediatric pulmonary hypertension in the Netherlands: epidemiology and characterization during the period 1991 to 2005. Circulation. 2011:124:1755-1764.

2. Ivy DD, Abman SH, Barst RJ, et al. Pediatric Pulmonary Hypertension. JACC. 2013;62(25,Suppl.D):117-126.

3. Fraisse A, Jais X, Schleich JM, et al. Characteristics and prospective 2-year follow-up of children with pulmonary arterial hypertension in France. Arch Cardiovasc Dis. 2010;103:66-74

4. Ghio S, Klersy C, Magrini G, et al. Prognostic relevance of the echocardio $\neg$ graphic assessment of right ventricular function in patients with idiopathic pulmonary arterial hypertension. Int $J$ Cardiol. 2010;140:272-278.

5. D'Alonzo GE, Barst RJ, Ayres SM, et al. Survival in patients with primary pulmonary hypertension. Results from a national prospective registry. Ann Intern Med. 1991;115:343-349.

6. Bristow MR, Zisman LS, Lowes BD, Abraham WT, Badesch DB, Groves BM, Voelkel NF, Lynch DM, Quaife RA. The pressure-overloaded right ventricle in pulmonary hypertension. Chest. 1998 Jul; 114(1 Suppl):101S-106S

7. Stojnic BB, Brecker SJ, Xiao HB, Helmy SM, Mbaissouroum M, Gibson DG. Left ventricular filling characteristics in pulmonary hypertension: a new mode of ventricular interaction. Br Heart J. 1992 Jul; 68(1):16-20.

8. Lopez L, Colan SD, Frommelt PC, et al. Recommendations for Quantification Methods During the Performance of a Pediatric Echocardiogram: A Report From the Pediatric Measurements Writing Group of the American Society of Echocardiography Pediatric and Congenital Heart Disease Council. J Am Soc Echocardiogr. 2010;23:465-495.

9. Dragulescu A, Mertens LL. Developments in echocardiographic techniques for the evaluation of ventricular function in children. Archives of Cardiovascular Disease. 2010;103:603-614.

10. Friedberg MK, Silverman NH. The systolic to diastolic duration ratio in children with hypoplastic left heart syndrome: a novel Doppler index of right ventricular function. J Am Soc Echocardiogr. 2007;20(6):749-755.

11. Raymond RJ, Hinderliter AL, Willis PW, Ralph D, Caldwell EJ, Williams W, Ettinger NA, Hill NS, Summer WR, de Boisblanc B, Schwartz T, Koch G, Clayton LM, Jöbsis MM, Crow JW, Long W. Echocardiographic predictors of adverse outcomes in primary pulmonary hypertension. J Am Coll Cardiol. 2002 Apr 3; 39(7):1214-1219.

12. Puwanant S, Park M, Popovic ZB, Tang W, Farha S, George D, Sharp J, Puntawangkoon J, Loyd JE, Erzurum SC, Thomas JD. Ventricular Geometry, Strain, and Rotational Mechanics in Pulmonary Hypertension. Circulation. 2010;121(2):259-266.

13. Amundsen $\mathrm{BH}$, Helle-Valle $\mathrm{T}$, Edvardsen $\mathrm{T}$, et al. Noninvasive myocardial strain measurement by speckle tracking echocardiography: validation against sonomicrometry and tagged magnetic resonance imaging. J Am Coll Cardiol. 2006;47:789-93.

14. Wang J, Sengupta PP, Miyazaki C, et al. Two-Dimensional Strain-A Doppler-Independent Ultrasound Method for Quantitation of Regional Deformation: Validation In Vitro and In Vivo. Journal of the American Society of Echocardiography. 2005;18:1247-53.

15. Hardegree EL, Sachdev A, Fenstad ER, Villaraga HR, Frantz RP, McGoon MD, Oh JK, Ammash NM, Connolly HM, Eidem BW, Pellikka PA, Kane GC. Impaired Left Ventricular Mechanics in Pulmonary Arterial Hypertension. Identification of a Cohort at High Risk. Circulation: Heart Failure. 2013;6:748-755.

16. Huez S, Vachiery JL, Unger P, Brimioulle S, Naeije R. Tissue Doppler imaging evaluation of cardiac adaptation to severe pulmonary hypertension. Am J Cardiol. 2007;100:1473-1478.

17. Delgado V, Ypenburg C, van Bommel RJ, Tops LF, Mollema SA, Marsan NA, Bleeker GB, Schalij MJ, Bax JJ. Assessment of Left Ventricular 
Dyssynchrony by Speckle Tracking Strain Imaging Comparison Between Longitudinal, Circumferential, and Radial Strain in Cardiac Resynchronization Therapy. J Am Coll Cardiol. 2008;51(20):1944-1952.

18. Asrar ul Haq M, Rudd N, Subiakto I, Barlis P, Anavekar NS. Speckle Tracking for Assessment of Left Ventricular Dyssynchrony. World Journal of Cardiovascular Diseases. 2014;4:149-155.
19. Dohi K, Onishi K, Gorcsan III J, López-Candales A, Takamura T, Ota S, Yamada N, Ito M. Role of Radial Strain and Displacement Imaging to Quantify Wall Motion Dyssynchrony in Patients with Left Ventricular Mechanical Dyssynchrony and Chronic Right Ventricular Pressure Overload. Am J Cardiol. 2008;101:1206-1212. 